\title{
Universal Fluctuations in Spectra of Disordered Systems at the Anderson Transition*
}

\author{
Isa Kh. ZhareKeshevl and Bernhard Kramer \\ 1 Institut für Theoretische Physik, Universität Hamburg, Jungiusstrasse 9, D-20355 Hamburg, \\ Germany
}

(21 February, 1995)

\begin{abstract}
Using the level-spacing distribution and the total probability function of the numbers of levels in a given energy interval we analyze the crossover of the level statistics between the delocalized and the localized regimes. By numerically calculating the electron spectra of systems of up to $32^{3}$ lattice sites described by the Anderson Hamiltonian it is shown that the distribution $P(s)$ of neighboring spacings is scale-independent at the metal-insulator transition. For large spacings it has a Poisson-like asymptotic form $P(s) \propto \exp (-A s / \Delta)$, where $A \approx 1.9$. At the critical point we obtain a linear relationship between the variance of the number of levels $\left\langle[\delta n(\varepsilon)]^{2}\right\rangle$ and their average number $\langle n(\varepsilon)\rangle$ within the interval $\varepsilon$. The constant of proportionality is less than unity due to the repulsion of the levels. Both $P(s)$ and $\left\langle[\delta n(\varepsilon)]^{2}\right\rangle$ are determined by the probability density $Q_{n}(\varepsilon)$ of having exactly $n$ levels in the energy interval $\varepsilon$. The distribution $Q_{n}(\varepsilon)$ at the critical point is found to be size-independent and to obey a Gaussian law near its maximum, where $n \sim\langle n\rangle$.
\end{abstract}

*to be appeared in Jpn. J. Appl. Phys., 34 (8A), 1995. 


\section{INTRODUCTION}

The statistical properties of the energy levels of disordered systems near the metalinsulator transition are presently attracting much attention. Due to Anderson localization, the one-electron states of systems with higher dimensionality $(d>2)$ are known to experience a crossover from delocalized to localized behavior. This metal-insulator transition (MIT) is induced by increasing the fluctuations of a random potential or by shifting the Fermi energy through the mobility edge at fixed disorder. [1] The statistics of the corresponding energy levels is very sensitive to the MIT and depends dramatically on the strength of disorder. In the insulating regime the levels are completely random, and their distribution obeys the Poissonian statistical laws of uncorrelated variables. On the other side of the MIT, in the metallic regime, correlations in the spectrum become stronger due to quantum-mechanical level repulsion. It was shown earlier [2, 3] that the level statistics in a disordered metal is governed by the random-matrix theory. [4 6

By using finite-size scaling arguments Shklovskii et al. [7] suggested that exactly at the MIT the distribution of spacings $P(s)$ between the neighboring energy levels exhibits critical behavior and is a scale-invariant function which differs considerably from both the Wigner surmise $P_{W}(s)=(\pi s / 2) \exp \left(-\pi s^{2} / 4\right)$ and the Poisson law $P_{P}(s)=\exp (-s)$, corresponding to the delocalized and the localized regime, respectively. Here $s$ is measured in units of the mean level spacing $\Delta$. Later the universal properties of $P(s)$ at the mobility edge were studied in some detail by using approximate analytical [8,9] and numerical methods. [10 12]

In this paper we present results of elaborate numerical investigations of the statistics of fluctuations in the energy spectra of three-dimensional (3D) disordered lattices near the MIT. Using the Anderson model we calculate very accurately not only the critical distribution $P(s)$ of neighboring spacings, but also the total probability density $Q_{n}(\varepsilon)$ of having $n$ levels within an energy interval $\varepsilon$. The latter contains more complete information about spectral correlations of higher orders. Both distributions exhibit a universal character at the critical disorder and become independent of the system size. We find that the variance 
of the number of the levels in an interval $\varepsilon$ as a function of mean number of levels is also scale-invariant, and obeys a linear relation $\left\langle\delta n^{2}\right\rangle=\kappa\langle n\rangle$ with $\kappa \approx 0.27$, when $\langle n\rangle \gg 1$.

\section{NEAREST-NEIGHBOR LEVEL SPACING DISTRIBUTION}

In order to calculate the spectrum we use the Hamiltonian corresponding to a tightbinding model with diagonal disorder, $H=\sum_{n} \varepsilon_{n} a_{n}^{\dagger} a_{n}+\sum_{n \neq m}\left(a_{n}^{\dagger} a_{m}+\right.$ c.c. $)$, where $a_{n}^{\dagger}\left(a_{n}\right)$ is the creation (annihilation) operator of an electron at a site $n$ in a simple cubic lattice. The random site energy $\varepsilon_{n}$ is distributed uniformly between $-W / 2$ and $W / 2$. The second sum involves only pairs of nearest sites $\{n, m\}$. The MIT in the center of the band corresponds to $W_{c} \approx 16.5$. [13]

The energy levels of cubes of various linear sizes from $L=5$ to 32 with periodic boundary conditions were calculated by direct diagonalization of the Hamiltonian using an optimized Lanczos algorithm. [12] Figure 11 displays the histogram of $P(s)$ calculated at the disorder corresponding to the critical point, $W_{c}$. The number of realizations was chosen so that the numbers of levels from central half part of the band were approximately $1.210^{7}, 1.210^{6}$ and $210^{5}$ for $L=5,12$ and 32, respectively. All of the obtained data, regardless of $L$, lie on the same curve, the critical $P(s)$, which is considerably different from both $P_{W}(s)$ and $P_{P}(s)$. However, for disorder $W \neq W_{c}$ the distribution $P(s)$ depends on the system size and exhibits scaling behavior near the MIT. [7] Indeed, for decreasing $W$ the distribution of spacings approaches the Wigner surmise, while for increasing $W$ it scales towards a Poissonian law. This crossover is apparently accompanied by the transition from the delocalized to the localized regime. The finite-size scaling properties of $P(s)$ allow determination of the critical exponent $\nu \approx 1.45$ and the disorder dependence of the scaling parameter, the correlation length $\xi(W)$. [11,12] We have also verified that the form of $P(s)$ exactly at the MIT does not depend on the width of the energy interval as long as the levels in this interval belong to the critical region, i.e. satisfy the condition $L<\xi=\left(\left|\varepsilon-\varepsilon_{c}\right| / \varepsilon_{c}\right)^{-\nu}$, where $\varepsilon_{c}$ is the mobility edge. 
Using a large number of realizations of the randomness we were able to analyze the asymptotic behavior of the critical $P(s)$ as shown in the inset of Fig. 11. For spacings $s>2$ the exponential tail of $P(s)$ is intermediate between the Gaussian and the Poissonian. It is well described by the relation $P(s)=\exp (-A s)$ with a numerical coefficient $A \approx 1.9$. One observes that this asymptotic decay deviates from the power-law $\ln P(s) \propto s^{1+1 / 3 \nu}$ which was recently found [8] by using the effective "plasma model" as defined by Dyson. [5]

\section{PROBABILITY OF $N$ LEVELS IN A GIVEN INTERVAL}

In order to analyze the correlations between several consecutive eigenvalues, one can study the probability that an energy interval of width $\varepsilon$ centered at a randomly chosen energy contains exactly $n$ levels. The distribution of this joint probability $Q_{n}(\varepsilon)$ is related to the $n$-level correlation function $R_{n}\left(\varepsilon_{1}, \varepsilon_{2}, \ldots, \varepsilon_{n}\right)$. It provides a more complete description of the level statistics than the two-point correlation function, [6] $R_{2}\left(\varepsilon_{1}-\varepsilon_{2}\right)$, which can be expressed in terms of the $n$-level spacing distribution $p(n, s), R_{2}(s)=\sum_{n=0}^{\infty} p(n, s)$. On the other hand, the probability of finding no levels $(n=0)$ inside the interval defines the nearest-neighbor spacing distribution, $P(s)=d^{2} Q_{0}(s) / d s^{2}$.

The results of the random-matrix theory for $Q_{n}(\varepsilon)$, which correspond to the metallic case, have been calculated numerically, [6] and were later expressed in an explicit analytical form [14] for the three universality classes of random Hamiltonians: orthogonal, unitary and symplectic. In the limit $\delta n \equiv|n-\varepsilon| \ll \varepsilon$ the distribution for the orthogonal ensemble is approximately Gaussian,

$$
\ln Q_{n}(\varepsilon) \propto-\frac{\pi^{2}}{4} \frac{\delta n^{2}}{\ln (8 \varepsilon /|\delta n|)+B}
$$

where $\varepsilon$ is measured in $\Delta$ and $B$ depends weakly on $\delta n$.

In the insulating regime the sequence of the levels is completely random due to the localization of the states, and therefore we have the usual Poissonian process,

$$
Q_{n}(\varepsilon)=\varepsilon^{n} \exp (-\varepsilon) / n !
$$


For example, if $n=0$ the Wigner surmise and the Poisson law give

$$
Q_{0}(\varepsilon)=1-\frac{2}{\sqrt{\pi}} \operatorname{Err}\left(\frac{\sqrt{\pi}}{2} \varepsilon\right), \quad Q_{0}(\varepsilon)=\exp (-\varepsilon)
$$

for the metallic and insulating regime, respectively. $\operatorname{Err}(x)$ is the error function.

It is of great interest to study how $Q_{n}(\varepsilon)$ changes from (11) to (A) with increasing disorder $W$, and to calculate its shape at the MIT. Our results suggest that the function $Q_{n}(\varepsilon)$ shows critical behavior near $W_{c}$. In Fig. 2 we show $Q_{n}(\varepsilon)$ corresponding to the critical point. Calculations for different system sizes at $W_{c}$ yield almost the same set of distributions independently of the number of levels $n=0,1,2, \ldots$. The width of $Q_{n}(\varepsilon)$ at the MIT is larger than in the metallic regime, since the level repulsion becomes less important. We note that for small fluctuations $(\delta n \ll \varepsilon) \ln Q_{n}(\varepsilon)$ is quadratic with respect to both $\varepsilon$ and $n$, similarly to (11).

Let us consider the quantity $I_{n}=\int_{o}^{\infty} Q_{n}(\varepsilon) d \varepsilon$. For the Gaussian orthogonal ensemble it is known from previous numerical simulations [6] that $I_{0} \approx 0.643, I_{1} \approx 0.922$, and $I_{n}$ converges to unity in the limit $n \rightarrow \infty$. For the Poissonian process (2) it is easy to see that $I_{n}=1$ for all $n$. At the MIT, when $W=16.5$, we obtained the following values of the integral: $I_{0}^{c} \approx 0.714, I_{1}^{c} \approx 0.960, I_{2}^{c} \approx 0.994, I_{3}^{c} \approx 0.998$, and also $\lim _{n \rightarrow \infty} I_{n}^{c}=1$. The latter set of constants is $L$-independent and characterizes the critical level statistics. We emphasize that $I_{n}$ changes with $W$ around the critical disorder $W_{c}$ according to a one-parameter scaling law, $I_{n}(W, L)=I_{n}^{c}+f(L / \xi(W))$, where the function $f(x)$ can be linearized in the vicinity of the critical point, $f(L, W) \propto\left(W-W_{c}\right) L^{1 / \nu}$. This allows determination of the critical exponent $\nu$ of the correlation length. Note that in searching for $\nu$ it is not necessary to choose a certain spacing $s_{o}$ as was done earlier when using $P(s)$. [11, 12]

\section{VARIANCE OF THE LEVEL NUMBER}

The width of the distribution $Q_{n}(\varepsilon)$ describes the rigidity of the spectrum, which is

defined by the variance of the level number, $\left\langle[\delta n(\varepsilon)]^{2}\right\rangle=\sum_{n=0}^{\infty}(n-\langle n\rangle)^{2} Q_{n}(\varepsilon)$, where $\langle n\rangle=\varepsilon$ 
is the average number of levels in a given interval $\varepsilon$. One can ask how dependence of the variance $\left\langle[\delta n(\varepsilon)]^{2}\right\rangle$ on the average $\langle n(\varepsilon)\rangle$ changes when the delocalized states transform into the localized ones.

It follows from the random-matrix theory [3, 6] that in the metallic regime the variance is given by the Dyson formula,

$$
\left\langle\delta^{2} n\right\rangle_{\mathrm{M}}=\frac{2}{\pi^{2}}(\ln \langle n\rangle+C), \quad 1 \ll\langle n\rangle \ll \hbar D / L^{2}
$$

where $C \approx 2.18$ and $D$ is the diffusion constant. By increasing the disorder $W$ the fluctuations of $\delta n$ are increased due to a weakening of level correlations. In the strongly localized regime the levels are not correlated, hence $\left\langle\delta^{2} n\right\rangle_{\mathrm{I}}=\langle n\rangle$. This is much larger than $\left\langle\delta^{2} n\right\rangle_{\mathrm{M}}$. Exactly at the MIT, the variance depends linearly on the average level number, [15] $\left\langle\delta^{2} n\right\rangle_{c}=\kappa\langle n\rangle$, as in the insulating limit. However the factor $\kappa$ is less than unity.

We calculated the dependence of the ratio $\left\langle\delta n^{2}\right\rangle /\langle n\rangle$ on the average number of levels within a given interval $\varepsilon$ for different lattice sizes $L$ at $W_{c}=16.5$ as shown in Fig. 3 . Obviously the ratio is not sensitive to a change of $L$. Such behavior results from the aboveFig. 3 mentioned universality of the probability density $Q_{n}(\varepsilon)$. One observes that the amplitude of the relative fluctuations of the number of levels $\left\langle\delta^{2} n\right\rangle /\langle n\rangle$ decreases very weakly with the energy, approaching the constant limit $\kappa \approx 0.27$. Thus, our results are consistent with the suggestion of proportionality between the variance and the average level number at large $\langle n\rangle$, 15 but deviate from the recently proposed power law, 9] $\left\langle\delta^{2} n\right\rangle /\langle n\rangle \propto\langle n\rangle^{-1 / 3 \nu}$. However, this power law can be considered in our calculations as the next higher-order correction to the leading constant term.

It was earlier conjectured [15] that $Q_{n}(\varepsilon)$ at the MIT is normally distributed $\propto$ $\exp \left[-(\varepsilon-n)^{2} / 2\left\langle\delta^{2} n\right\rangle_{c}\right]$ for fixed $\varepsilon \gg n$. After substituting $k \varepsilon$ for the variance and doubly differentiating, for $n=0$ one recovers the Poissonian asymptotic form of the critical $P(\varepsilon) \propto \exp (-\varepsilon / 2 \kappa)$. 


\section{CONCLUSIONS}

We have discussed the statistics of the spectral fluctuations in the 3D systems at the disorder-induced metal-insulator transition. By directly diagonalizing the Anderson Hamiltonian the nearest-neighbor level spacing distribution $P(s)$ and the probability $Q_{n}(\varepsilon)$ of finding exactly $n$ eigenvalues in an energy interval of width $\varepsilon$ were numerically calculated. We confirmed that the critical $P(s)$ is $L$-independent and has a Poissonian like asymptotic form at large $s$, but with stronger decay than that in the insulating regime. One expects that the critical $Q_{n}(\varepsilon)$ which describes the statistical fluctuations in the discrete spectrum exhibits also complete scale-invariance in analogy with the universality of the critical $P(s)$. Our results show that the distributions $Q_{n}(\varepsilon)$ at the MIT are well described by Gaussians both for small fluctuations $\delta n \ll \varepsilon$ and also when $n \ll \varepsilon$, as in the metallic limit. We also analyzed the variance of the number of states $\left\langle\delta^{2} n\right\rangle$ in a given interval as a function of mean level number $\langle n\rangle$. At the critical point this function was found to be independent of the system size and to have a leading linear term, $\left\langle\delta^{2} n\right\rangle_{c}=0.27\langle n\rangle$ for $n \gg 1$. To summarize, our results indicate that the statistics of the energy levels near the Anderson transition constitute an infinite set of scaling variables $I_{n}$ which can be used to characterize the mobility edge by one-parameter scaling laws.

\section{ACKNOWLEDGMENTS}

The authors thank B. I. Shklovskii for discussions. Financial support from DAAD during the stay of I. Kh. Zh. at the University of Hamburg is gratefully acknowledged. 


\section{REFERENCES}

$\S \quad$ Permanent address: Department of Physics, Kazakh State University, Almaty, Kazakhstan. E-mail address: isa@physnet.uni-hamburg.de

[1] For review, see B. Kramer and A. MacKinnon: Rep. Prog. Phys. 56 (1994) 1469 and references therein.

[2] K. B. Efetov: Adv. Phys. 32 (1983) 53.

[3] B. L. Altshuler and B. I. Shklovskii: Sov. Phys. JETP 64 (1986) 127.

[4] E. P. Wigner: Ann. Math. 62 (1955) 548.

[5] F. J. Dyson: J. Math. Phys. 3 (1962) 140.

[6] M. L. Mehta: Random Matrices (Academic Press, Boston, 1991).

[7] B. I. Shklovskii, B. Shapiro, B. R. Sears, P. Lambrianides and H. B. Shore: Phys. Rev. B 47 (1993) 11487.

[8] A. G. Aronov, V. E. Kravtsov and I. V. Lerner: JETP Lett. 59 (1994) 40.

[9] V. E. Kravtsov, I. V. Lerner, B. L. Altshuler and A. G. Aronov: Phys. Rev. Lett. 72 (1994) 888 .

[10] S. N. Evangelou: Phys. Rev. B 49 (1994) 16805.

[11] E. Hofstetter and M. Schreiber: Phys. Rev. B 49 (1994) 14726.

[12] I. Kh. Zharekeshev and B. Kramer: to be published in Phys. Rev. B (1995).

[13] A. Mac Kinnon and B. Kramer: Z. Phys. B 53 (1983) 1.

[14] M. M. Fogler and B. I. Shklovskii: to be published in Phys. Rev. Lett. (1995).

[15] B. L. Altshuler, I. Kh. Zharekeshev, S. A. Kotochigova and B. I. Shklovskii: Sov. Phys. JETP 67 (1988) 625. 


\section{FIGURES}

FIG. 1. Level spacing distribution $P(s)$ at the MIT for various system sizes $L$. Curves: Wigner and Poisson distributions. Inset shows the tail of $P(s)$. Straight line is best fit. Dotted line: the power-law asymptotic [8] $\ln P(s) \propto s^{1+1 / 3 \nu}$.

FIG. 2. Critical probability distribution $Q_{n}(\varepsilon)$ for different $n$.

FIG. 3. Variance of the level number $\left\langle\delta n^{2}\right\rangle$ as a function of the average $\langle n\rangle$ for various $L$. Solid line: Dyson result (4). 


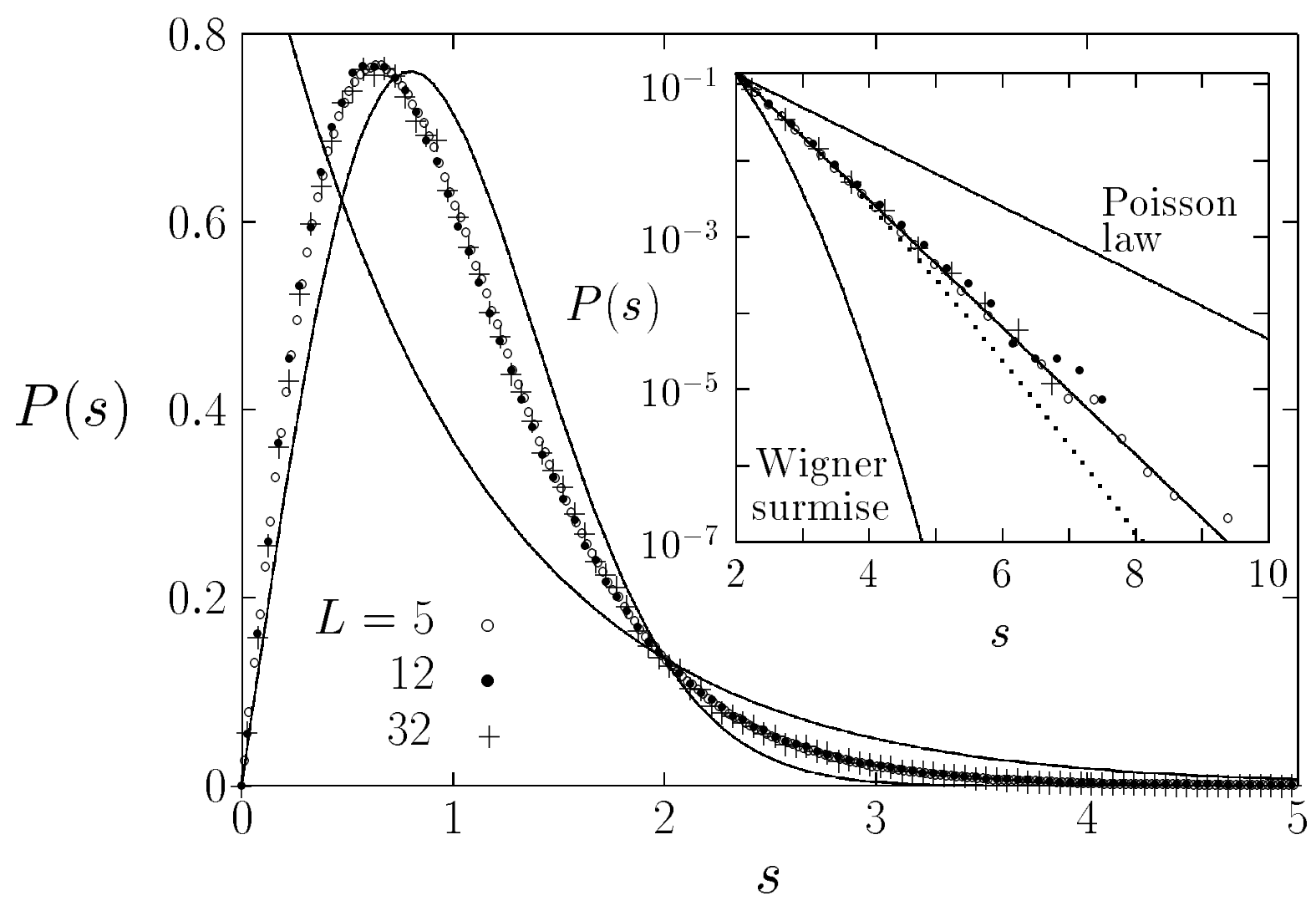




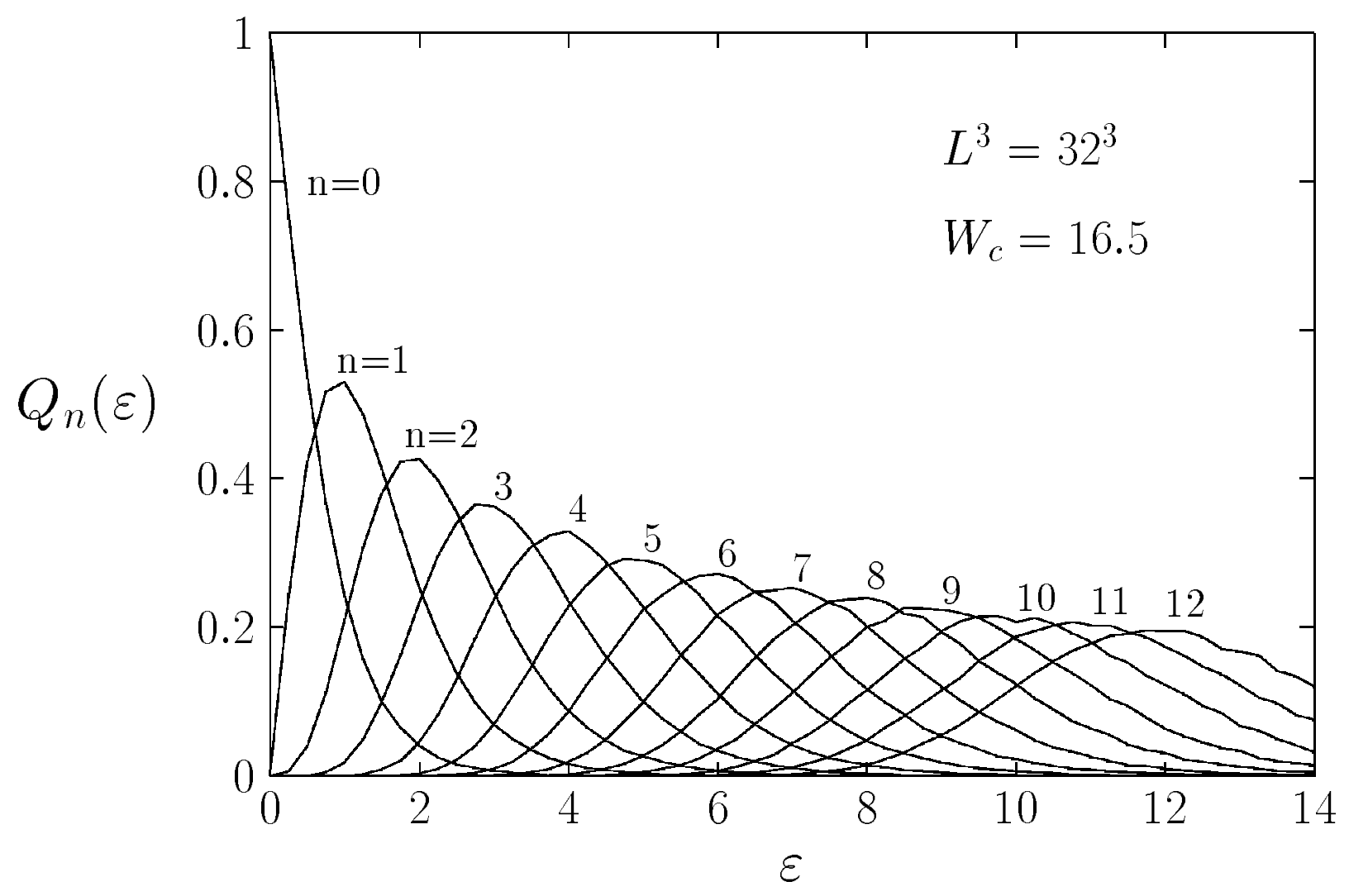






